\title{
FRET-CLSM and double-labeling indirect immunofluorescence to detect close association of proteins in tissue sections
}

\author{
Peter König ${ }^{1, *}$, Gabriela Krasteva ${ }^{1, *}$, Claudia Tag ${ }^{1}$ Inke R König ${ }^{2}$, Christoph Arens ${ }^{3}$ \\ and Wolfgang Kummer ${ }^{1}$ \\ ${ }^{1}$ Institut für Anatomie und Zellbiologie, Justus-Liebig-Universität Giessen, Giessen, Germany; ${ }^{2}$ Institut für \\ Medizinische Biometrie und Statistik, Universität zu Lübeck, Lübeck, Germany and ${ }^{3}$ Hals-Nasen- \\ Ohrenklinik, Universitätsklinikum Giessen und Marburg-Standort Giessen, Giessen, Germany
}

\begin{abstract}
It is pivotal to identify protein-protein interaction in situ to understand protein function. Conventional methods to determine the interaction of proteins destruct tissue or are applicable to cell culture only. To identify association of proteins in cells in tissue, we adapted indirect double-labeling immunofluorescence and combined it with conventional confocal laser scanning microscopy (CLSM) to measure fluorescence resonance energy transfer (FRET). As a model system, we chose caveolin-1 $\alpha$ and caveolin-2, two major components of endothelial caveolae, and examined their interaction in the endothelium of vessels in fixed tissues of laboratory animals and human glomus tumors. Several methodological aspects were examined. Measuring the absolute increase in fluorescence (AIF) was superior compared to determining the relative FRET efficiency, because it is more robust against small increases of fluorescence during measurements that results from unavoidable minimal crossreactivity of the secondary antibodies. Both, sequential and simultaneous incubation of secondary antibodies result in robust and reliable increases in $\Delta \mathrm{IF}$. If incubated sequentially, however, the acceptor-labeled secondary antibody should be applied first. The size of the secondary reagent $\left(F\left(a b^{\prime}\right)_{2}\right.$ vs whole antibody) has no major influence. In conclusion, CLSM-FRET can measure close spatial association of proteins in situ and can be applied to human surgical material.
\end{abstract}

Laboratory Investigation (2006) 86, 853-864. doi:10.1038/labinvest.3700443; published online 19 June 2006

Keywords: fluorescence resonance energy transfer; FRET; double-labeling immunohistochemistry; caveolin; tissue sections; human material

To understand the function of a protein, it is pivotal to identify interacting proteins that modulate its function and to determine where and under what circumstances they interact within a cell. Many techniques have been developed to identify proteinprotein association in cell culture and in tissue homogenates. Such approaches, however, provide only very limited information about the situation in situ. Cell culture gives detailed information only for a system reduced greatly in complexity, and tissue homogenates give no information in which cell type the interaction occurs. Therefore, it is desirable to detect a close spatial association of proteins in a

Correspondence: Dr P König, MD, Institut für Anatomie und Zellbiologie, Justus-Liebig-Universität Giessen, Aulweg 123, Giessen 35385, Germany.

E-mail: peter.koenig@anatomie.med.uni-giessen.de

* These authors contributed equally to this study.

Received 21 December 2005; revised and accepted 17 April 2006;

Published online 19 June 2006 given cell within a tissue, including identification of the cellular compartment in which it occurs. A simple method to study the localization of two proteins in tissue is double-labeling immunohistochemistry on tissue sections. At the conventional light microscopic level, the maximum spatial resolution is about $200 \mathrm{~nm}^{1}$ which is not sufficient to determine if proteins are located close enough to allow interaction. Fluorescence resonance energy transfer (FRET) is a nonradiative transfer of energy between two fluorophores, an energy-donating fluorophore called the donor, and an energy-accepting fluorophore called the acceptor. ${ }^{2,3}$ Since the efficiency of energy transfer is reduced by the sixth order of magnitude of the distance between these fluorophores, ${ }^{2}$ efficient energy transfer between two fluorophores occurs for most fluorophore pairs only if they are $<10 \mathrm{~nm}$ apart. This effect can be exploited to detect close spatial association of proteins by labeling them with fluorophores. Direct labeling of two proteins, either directly or by genetic 
means, alters the protein itself and/or its endogenous level in the cell. This may not reflect the situation found in situ. Furthermore, these techniques are not applicable to human material and, hence, do not allow analysis of pathological specimens. An easy and affordable method to label endogenous proteins with fluorescent dyes in tissue sections that can be applied to human material is immunofluorescence. Indeed, direct and indirect immunofluorescence have been successfully used to detect protein-protein interactions in cell culture. ${ }^{3}$ Direct labeling of small amounts of antibodies with fluorophores can, in principle, be done with commercially available kits but most commercially available antibodies are mixed with substantial amounts of other proteins that interfere with the labeling reaction which limits its practical application.

So far two studies have employed indirect immunofluorescence for FRET experiments in tissue sections. One detected spatial association of proteins in pathologic protein aggregates not reflecting a physiologic concentration of proteins ${ }^{4}$ and the other used a sophisticated and not generally available two photon setup. ${ }^{5}$

The goal of our study was to develop a method to use indirect immunofluorescence to detect a close spatial association of two proteins whose expression is not pathologically altered in tissue sections using a conventional confocal laser scanning microscopy (CLSM). As a model system we chose to examine the interaction of caveolin- $1 \alpha$ (cav-1 $1 \alpha)$ and caveolin-2 (cav-2), two major proteins of caveolae that are known to form hetero-oligomers. ${ }^{6}$ Since the transport of cav-2 to caveolae is dependent on the coexpression of cav- $1,{ }^{7,8}$ it is safe to assume that both proteins interact also in caveolae and are a valid model to test the applicability of doublelabeling immunohistochemistry and FRET. Indeed, we were able to detect a FRET signal in endothelial cells using indirect immunofluorescence in tissue sections of specimens obtained from a laboratory animal (rat) under controlled conditions. We could further prove that the technique is also applicable to tissue sections from human tissue that was retrieved through surgical procedures.

\section{Materials and methods}

\section{Chemicals}

All chemicals were obtained from Sigma, Deisenhofen, Germany, unless otherwise stated.

\section{Tissue Preparation}

Wistar rats of either sex $(n=16)$ were killed by inhalation of isoflurane (Abbott, Wiesbaden, Germany). The thorax was opened and the lungs were inflated via the trachea using a 50\% OCT compound
(Sakura, Zoeterwoude, The Netherlands) in phosphate-buffered saline (PBS). Then, the thoracic organs were jointly removed, oriented in OCT compound on a piece of filter paper, and snap frozen in melting isopentane.

\section{Double-Labeling Immunohistochemistry}

Different incubation protocols were used and evaluated for their suitability for further FRET analysis. The following protocol was used and subsequently modified:

Ten micrometer thick sections were cut using a Leica CM-1900 cryostat (Leica, Bensheim, Germany), mounted on slides and subsequently dipped for $10 \mathrm{~min}$ in ice-cold acetone. Then, sections were incubated for $1 \mathrm{~h}$ at room temperature with $5 \%$ normal goat serum and $5 \%$ bovine serum albumin in PBS.

Then, sections were incubated overnight with rabbit cav- $1 \alpha$ antibody (1:400; Santa Cruz Biotechnology, Hamburg, Germany) together with a monoclonal mouse cav-2 antibody (1:200, Transduction Laboratories, Lexington, KY, USA) as mixture in PBS with normal salt concentration $(0.9 \% \mathrm{NaCl}$, PBS) or doubled salt concentration $(1.8 \% \mathrm{NaCl})$. After washing with PBS, the sections were incubated for $1 \mathrm{~h}$ with donkey anti-mouse IgG Cy3conjugated antibody $(1: 1000)$ and donkey antirabbit IgG Cy5-conjugated antibody (1:50) that both were crossadsorbed against rat serum proteins as well as serum proteins from the species of the other primary antibody (both from Dianova, Hamburg, Germany). After the final washing step, sections were fixed for $10 \mathrm{~min}$ in $4 \%$ paraformaldehyde in $0.1 \mathrm{M}$ phosphate buffer, $\mathrm{pH}$ 7.4, and washed three times in PBS. This fixation step proved to extend the stability of the labeling. Subsequently, the sections were coverslipped with carbonate-buffered glycerol, $\mathrm{pH}$ 8.6, or Mowiol 4-88, $\mathrm{pH} 8.6$, and stored at $4^{\circ} \mathrm{C}$ until evaluation. No differences were noted between the two mounting media with regard to bleaching of Cy5 and FRET signal. Coverslips of moviol-embedded sections, however, are less prone to dislocation since moviol hardens.

Slides that were incubated with a specific combination of antibodies were washed in separate cuvettes to prevent diffusion and subsequent binding of small amounts of primary antibody from other sections. This procedure was applied to all incubations.

The following modifications of the protocol were applied (sections from $n=4$ animals each):

1. To verify that the measured FRET signal was not originating from crossreactivity of secondary antibodies, the cav- 2 or the cav- $1 \alpha$ antibody, respectively, was omitted.

2. To evaluate if minimal amounts of bound donorlabeled antibodies still give a FRET signal the following incubation scheme was applied: The 
cav- $1 \alpha$ antibody was labeled using donkey antirabbit IgG Cy5-conjugated antibody (1:50) simultaneously with donkey anti-rabbit IgG Cy3-conjugated antibody that was serially diluted from $1: 2000$ to $1: 32000$.

3. To assess if the sequence of the application of the secondary antibodies influences the intensity of the FRET signal, the order of incubation of the secondary reagents was reversed.

4. To assess if the size of the secondary reagent influences the amount of the FRET signal, the Cy5-conjugated anti-rabbit IgG antibody was replaced with a donkey anti-rabbit IgG Cy5conjugated $\mathrm{F}\left(\mathrm{ab}^{\prime}\right)_{2}$ fragment (Dianova).

5. To assess if sequential application of antibodies is superior to simultaneous application, secondary antibodies were either applied simultaneously or sequentially with a washing step in between.

As further control, we performed a FRET experiment with cav- 2 and the $\alpha 10$ nicotinic acetylcholine receptor subunit that is expressed in heart ${ }^{9}$ and has been described in a subset of endothelial cells ${ }^{10}$ but, as all other nicotinic receptor subunits, has not been described as an interacting partner of cav-2. To detect the $\alpha 10$ nicotinic acetylcholine receptor subunit, we used a rabbit polyclonal antibody directed against the amino acids $404-417$ of the protein. ${ }^{11}$ For the detection, the same secondary reagents were used as in the cav-1-2 experiments.

To assess if we still detect an interaction between both cav isoforms if donor and acceptor label are switched, we labeled the cav-2 antibody with a donkey anti-mouse IgG Cy5-conjugated antibody (Dianova) and the cav-1 antibody with a donkey anti-rabbit IgG Cy3-conjugated antibody (Chemicon, Hampshire, UK).

\section{Application to Human Pathological Material}

To assess if the method is applicable to human tissue that was removed during surgical procedures, sections from glomus tumors that were Zamboni fixed $1.85 \%$ formaldehyde and $15 \%$ saturated picric acid solution in $0.1 \mathrm{M}$ phosphate buffer, $\mathrm{pH}$ $7.4)$ and cryoprotected $(18 \%$ sucrose in $0.1 \mathrm{M}$ phosphate buffer, $\mathrm{pH}$ 7.4) were incubated as described above using the optimized protocol. The glomus tumors were obtained during the years 1997-2003 $(n=4)$, anonymized, and stored at $-80^{\circ} \mathrm{C}$ until use.

\section{Measurement of FRET}

FRET was measured with the acceptor bleaching method using a Leica CLSM TCS SP2 AOBS equipped with an oil immersion objective $\times 63$, 1.4. NA (Leica, Bensheim, Germany). The following settings were used throughout the experiments: excitation for Cy3: HeNe laser with $543 \mathrm{~nm}$ (51\% intensity), excitation for Cy5: HeNe laser $633 \mathrm{~nm}$
(20\% laser intensity); detection wavelength for Cy3: 550-602 nm, detection wavelength for Cy5: $642-705 \mathrm{~nm}$. The pinhole was set to 3 airy units. Photomultiplier tube (PMT) settings were between 480 and $591 \mathrm{~V}$ to measure in the linear range of the PMT. Gain and laser intensity for detection were held constant during an experiment. The two fluorophores were detected sequentially in a line-by-line mode. Thus, only the adequate laser for each fluorophore was switched on to prevent the excitation of the other fluorophore and no crosstalk between both channels was detected even at higher PMT settings than the ones used for FRET experiments.

FRET was assessed in endothelial cells in tissue sections of the rat mediastinum including the heart.

The measurements were performed with the FRET acceptor bleaching application of the CLSM software as follows: Before and after bleaching, images in the Cy3(donor)- and the Cy5(acceptor) channel were acquired in the line-by-line sequential mode with three averaging steps to reduce noise. Cy5 was bleached 5-10 times (1.28 s/scan) with highest possible zoom $(\times 32)$ with $100 \%$ laser power of the $633 \mathrm{~nm}$ HeNe laser line. The number of bleaching steps that were sufficient to bleach Cy5 was determined at the beginning of each experiment and was held constant throughout. FRET was quantified by measurement of the fluorescence intensity in the Cy3(donor) channel in the bleached area before and after bleaching. Since the squaresized bleaching area also included the vascular lumen or other nonlabeled parts of the tissue, a second measurement was performed outlining the endothelial cell using the freehand region of interest (ROI) encircling tool of the software.

To assess changes during the measurements that were not due to FRET, four rectangular sentinel ROIs of approximately the same size as the bleached area were measured adjacent to the bleached area. Measurements were discarded if changes in FRET signal in these ROIs were obvious. Six measurements were performed for each animal and experimental condition.

\section{Statistical Analysis}

The resulting change in fluorescence intensity was depicted as (a) FRET efficiency $\left(\right.$ FRET $_{\text {eff }}$ ) expressed as percentage of fluorescence increase calculated by: FRET $_{\text {eff }}=\left(\left(I_{\mathrm{DA}}-I_{\mathrm{DB}}\right) / I_{\mathrm{DA}}\right) \times 100$, and (b) change of fluorescence intensity (absolute increase in fluorescence $(\Delta \mathrm{IF}))=I_{\mathrm{DA}}-I_{\mathrm{DB}}$, where $I_{\mathrm{DA}}$ is the donor intensity after bleaching and $I_{\mathrm{DB}}$ the donor intensity before bleaching.

To compare two experimental conditions and their controls, all groups of the experiment were compared by Kruskal-Wallis test. If the resulting $P$-value was $<0.05$, two individual groups were compared by Mann-Whitney tests in a hierarchical order. $^{12}$ Specifically, we firstly tested experimental 
conditions vs respective control conditions, then two experimental conditions against each other. The comparison was rated significant if $P<0.05$ and hierarchical testing of individual groups was stopped if $P>0.05$ to correct for multiple testing.

To assess if $\triangle \mathrm{IF}$ or $\mathrm{FRET}_{\text {eff }}$ is superior to discriminate between FRET experiments and controls, their receiving operator characteristics (ROC) curves were generated and the areas under each curve were compared using the method of Hanley. ${ }^{13}$

To test whether the size of the secondary antibody and the mixing or sequential application of both secondary antibodies determines the intensity of the FRET-signal, we varied the size of the secondary reagent using a Cy5-labeled $\mathrm{F}\left(\mathrm{ab}^{\prime}\right)_{2}$ fragment instead of a Cy5-labeled whole IgG molecule as well as sequential application of both secondary antibodies vs mixing antibodies. To allow for a possible interaction between these factors, we performed an analysis of variance analyzing $\Delta \mathrm{IF}$ depending on the factors (a) size of antibody, (b) mode of application and (c) their interaction.

All statistical analysis was performed using SPSS versions 11.5.1 or 12.0 (SPSS GmbH Software, Munich, Germany) except for ROC curve analysis which was performed using MedCalc version 8.1.0.0 (MedCalc Software, Mariakerke, Belgium).

\section{Results}

Detection of FRET between cav-1 $\alpha$ and cav-2 in Tissue Sections and Negative Controls

Using standard indirect immunofluorescence technique, FRET was robustly detected in endothelial cells in tissue sections of the rat mediastinum by bleaching the acceptor (Cy5) and detecting the increase in donor (Cy3) fluorescence. A doublelabeling of cav-1 and cav-2 is shown in Figure 1 and a FRET experiment is shown in Figure 2. To test for crossreactivity of secondary antibodies, one of the primary antibodies was omitted but both secondary antibodies were applied (Figure 3). If that primary antibody was omitted which was normally detected with a Cy5-labeled secondary antibody, no FRET signal was obtained (median: $\mathrm{FRET}_{\text {eff }} 0 \%, \Delta \mathrm{IF}$ -0.56) and there was no statistically significant difference compared to a single incubation with one primary antibody labeled with Cy3-conjugated secondary antibody (median: $F_{R E T}$ eff $0 \%, \Delta \mathrm{IF}-1.00$ ). Since we measured a negative $\Delta I F$ in this experiment, a small decrease in Cy3 fluorescence either due to the bleaching procedure or occurring during the image acquisition was present, indicating that the FRET signal is underestimated. In contrast, omission of that primary antibody which was normally labeled with Cy3-conjugated secondary antibody, resulted in detectable FRET signal when both secondary antibodies were applied (median: FRET $_{\text {eff }} 10.94 \%, \Delta \mathrm{IF} 2.99$ ) although $\Delta \mathrm{IF}$ and $\mathrm{FRET}_{\text {eff }}$ were significantly lower than in the experimental group. Swapping the donor and acceptor label still resulted in a detectable FRET signal between cav-1 and cav-2 although the signal was weaker (Figure 4). No statistically significant difference in FRET signal was detected between the $\alpha 10$ nicotinic acetylcholine receptor and cav-1 and the negative control (Figure 5). As a false-positive source for FRET we could occasionally detect changes in ROIs outside the bleached area that were not due to FRET but to movements of the specimen in $z$-direction that resulted from air movements of the air-conditioning system or from opening the door of the microscope room. Since these changes could be detected by our sentinel ROIs that were not bleached (cf. Figure 2), these false-positive experiments could be readily identified and were excluded from further analysis.

\section{Dilution Series of Cy3-Conjugated Secondary Antibody}

To assess if crossreactivity of the secondary antibodies accounts for the FRET signal seen in negative controls, we simulated crossreactivity by detecting one primary antibody with two secondary anti-
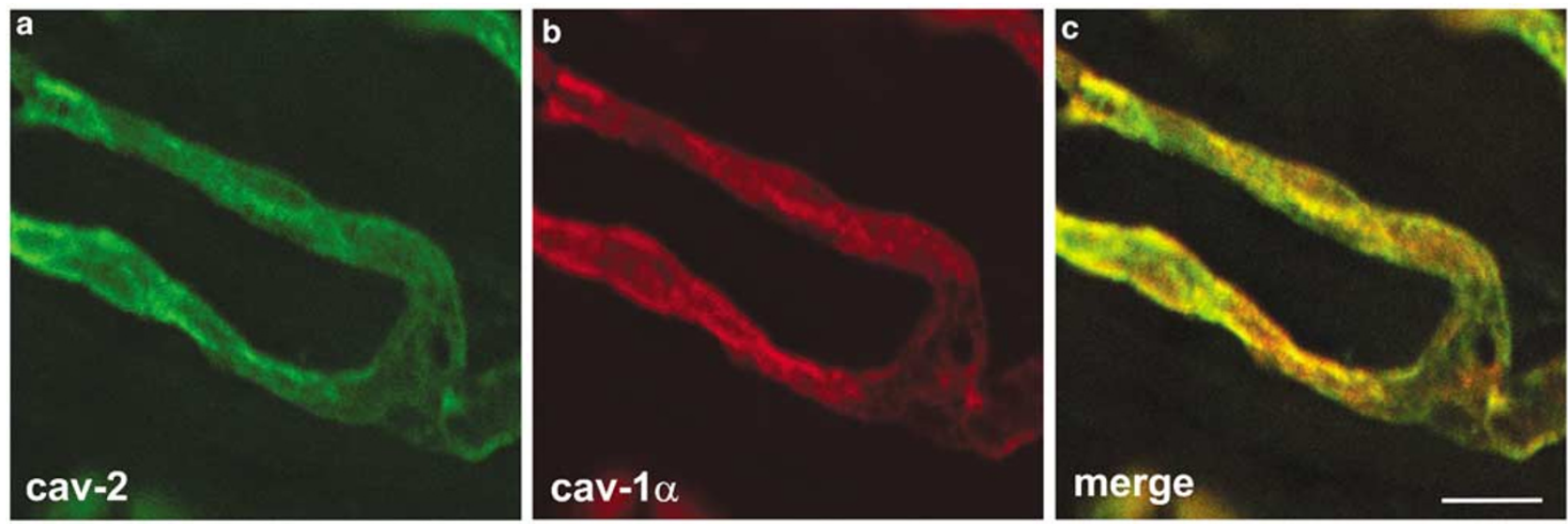

Figure 1 Double labeling of cav-1 and cav-2 in the rat heart. Immunolabeling indicates high amount of colocalization of cav-1 (a) and cav-2 (b) (see yellow color in the merged image, c). Bar $=10 \mu \mathrm{m}$. 
donor (Су3)
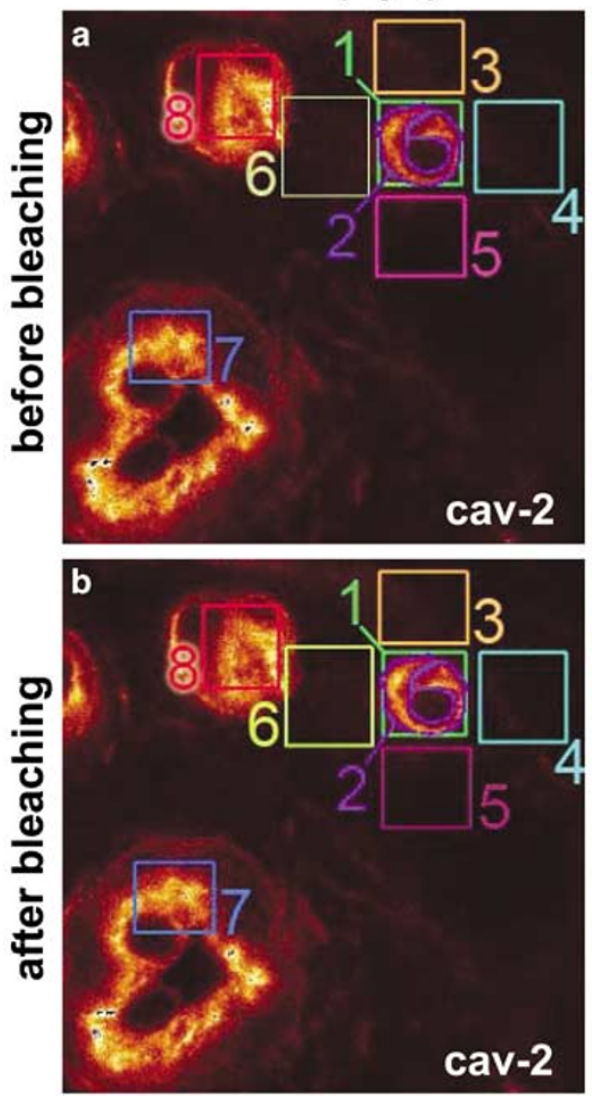

acceptor (Cy5)
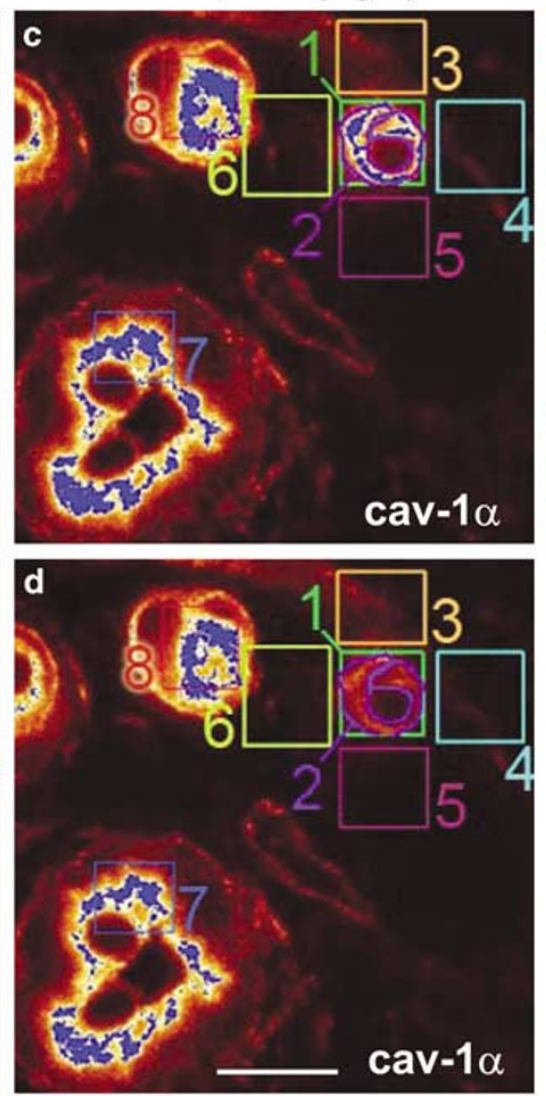

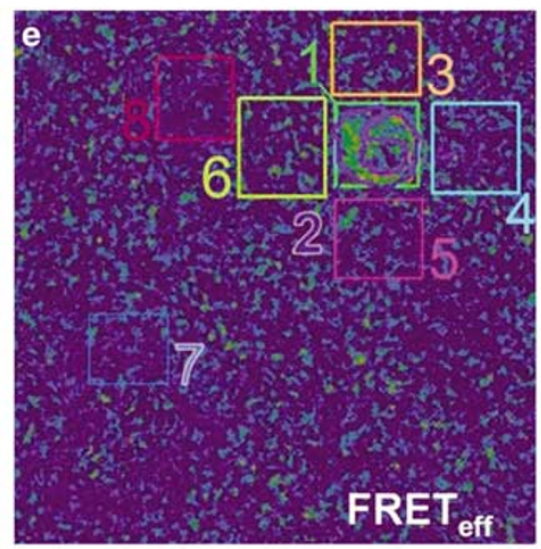

f ROI

$\Delta \mathrm{IF}$

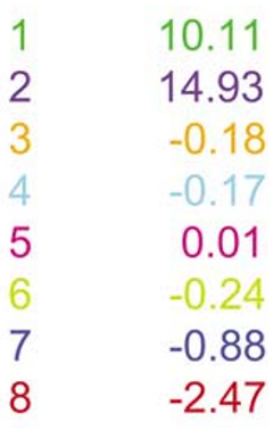

Figure 2 Detection of close association of cav-1 and cav-2 in endothelial cells by indirect immunofluorescence and FRET in tissue sections of the rat mediastinum. Images of donor (cav-2 labeled with Cy3-conjugated secondary antibody (a and b)) and acceptor (cav-1 $\alpha$ labeled with Cy5-conjugated secondary antibody (c and d)) fluorescence. Cy5 was bleached in region of interest (ROI) 1 (compare ROI 1 c

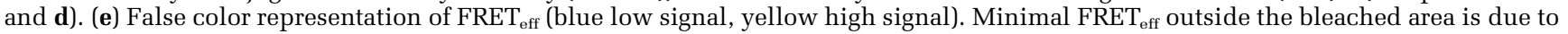
noise during acquisition of the images. (f) Changes in $\Delta \mathrm{IF}$ for each ROI. ROI 1: bleached area (compare (c and d)). ROI 2: freehandencircled region of endothelial cell in bleached area. ROI 3-8: control areas outside the bleached area. Bar $=10 \mu \mathrm{m}$.

bodies labeled with Cy3 and Cy5, respectively. Since both secondary antibodies used in this experiment bind to the same primary antibody (ie rabbit IgG) this situation is comparable to crossreactivity. The Cy3-labeled antibody was serially diluted to simulate lower amounts of bound crossreacting antibody. A high FRET signal up to $30 \%$ (median 21.98\%) could be detected (Figure 6) at the highest concentration of Cy3-labeled antibody used. Serial dilution of the Cy3-conjugated secondary reagent (donor) reduced FRET. At the highest dilution of 1:32000 (Figure 6) still FRET eff $_{\text {up }}$ to $12 \%$ (median 3.29\%) was detected although Cy3 fluorescence was no longer microscopically visible and the $\Delta \mathrm{IF}$ was small (median 0.82). This indicates that binding of minute amounts of Cy3-labeled antibody that results from minimal crossreactivity, can result in considerable $\mathrm{FRET}_{\text {eff }}$ although the underlying $\triangle \mathrm{IF}$ is minimal.

\section{Comparison of Suitability of $\Delta \mathrm{IF}$ and $\mathrm{FRET}_{\text {eff }}$ to Detect FRET in Tissue Sections}

Since high $\mathrm{FRET}_{\text {eff }}$ can result from small increases in $\Delta \mathrm{IF}$ that might result from crossreactivity of the donor-labeled secondary antibody if the initial donor fluorescence intensity is small, we assessed whether $\triangle \mathrm{IF}$ is a better parameter to detect FRET in indirect immunofluorescence. For this purpose the ability of $\mathrm{FRET}_{\text {eff }}$ and $\triangle \mathrm{IF}$ to discriminate the control vs the experimental group was assessed by ROC curves (Figure 7). The difference between both ROC curves was highly significant $(P<0.001)$ indicating that $\Delta \mathrm{IF}$ is superior to discriminate between FRET experiments vs control experiments (Figure 7).

\section{Incubation Sequence of Secondary Antibodies}

Examination of the sequence of the application of the secondary antibodies revealed that incubation with the Cy5-conjugated secondary antibody (acceptor) before incubation of Cy3-conjugated secondary antibody (donor) resulted in higher FRET signal (median: $\mathrm{FRET}_{\text {eff }} 6.64 \%, \Delta \mathrm{IF}$ 4.76) compared to incubation with Cy3-conjugated secondary antibody first (median: FRET $_{\text {eff }} 4.61 \%, \Delta \mathrm{IF} 2.19$; Figure 8). 


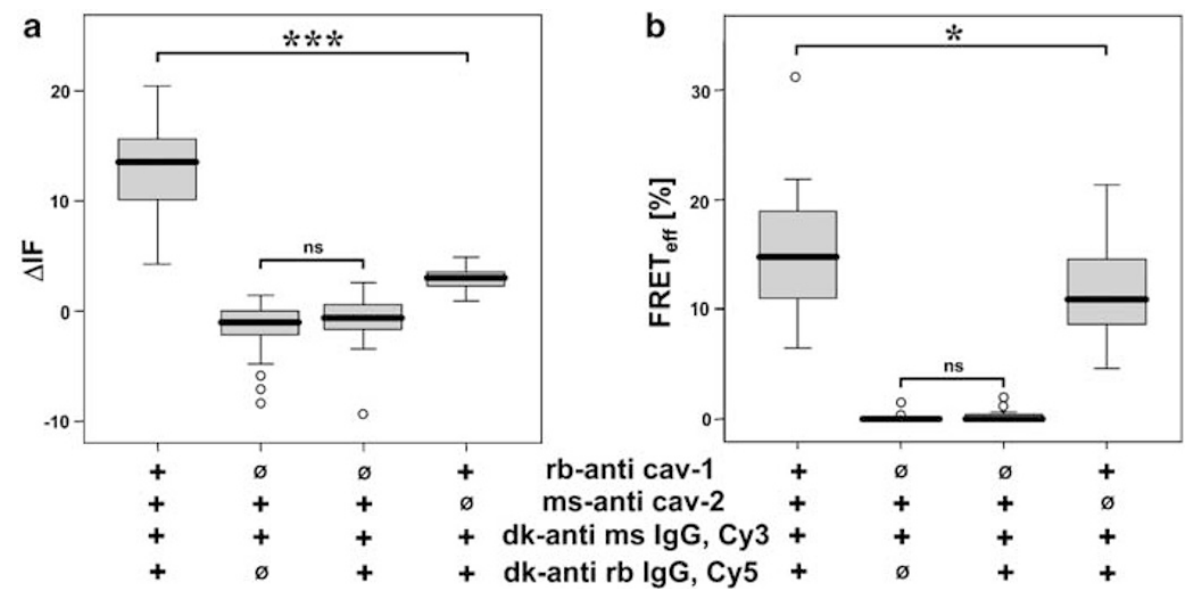

Figure 3 Control experiments. Experiment with both primary and secondary antibodies compared to omission of one of the primary antibodies and a single labeling with cav-2 and a Cy3-conjugated secondary antibody (sections from four animals with six measurements each). (a) Changes in $\Delta \mathrm{IF}$, (b) same data expressed in $\mathrm{FRET}_{\text {eff. }}{ }^{*}=P<0.05$, ${ }^{*} * *=<0.001$, NS $=P>0.05$. Boxplots: percentiles 0 , 25, median $75,100, \mathrm{O}=$ extreme values of data set. Secondary antibodies were sequentially incubated (Cy5-conjugated reagent first).

a

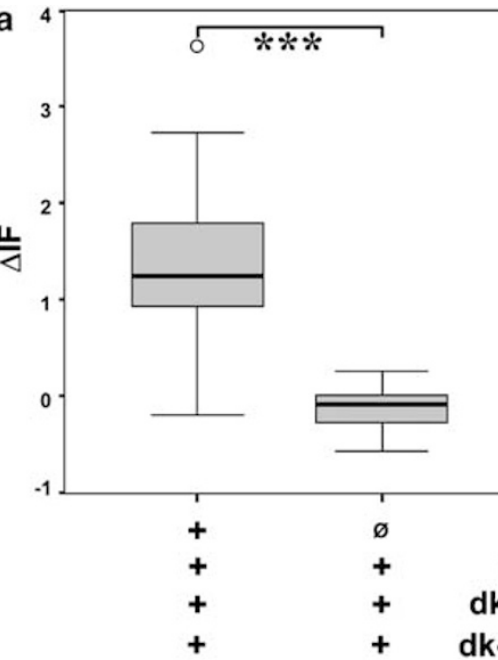

b

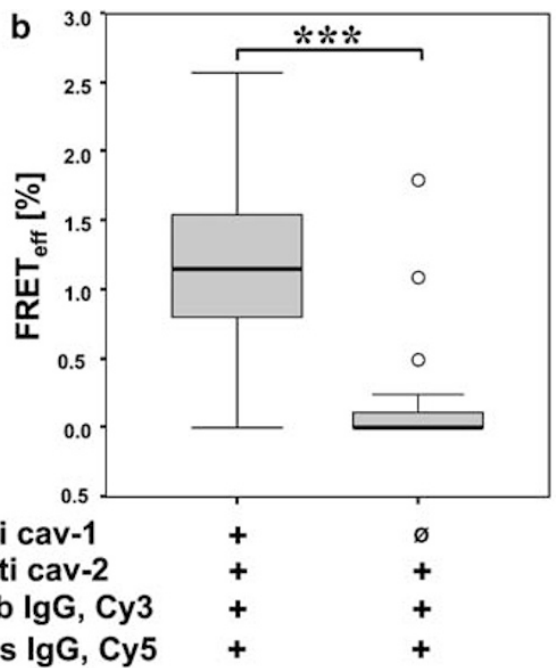

Figure 4 Swapping of secondary antibodies. Cav-1 was labeled with Cy3-conjugated secondary antibody and cav-2 was labeled with a Cy5-conjugated secondary antibody (sections from four animals with six measurements each). (a) Changes in $\Delta I F$, (b) same data expressed in FRET $_{\text {eff }},{ }^{* *}=P<0.001$. Boxplots: percentiles 0,25 , median $75,100, \mathrm{O}=$ extreme values of data set. Secondary antibodies were sequentially incubated (Cy5-conjugated reagent first).

\section{Sequential Application vs Simultaneous Application of Secondary Antibodies and Use of $F\left(a^{\prime}\right)_{2}$ Fragments vs Whole Secondary Antibodies}

To assess if the size of the secondary antibody determines the intensity of the FRET signal, we tested if the use of a Cy5-labeled $\mathrm{F}\left(\mathrm{ab}^{\prime}\right)_{2}$ fragment instead of a Cy5-labeled whole IgG molecule resulted in higher FRET signal. In addition, we compared if the sequential application of both secondary antibodies is superior to mixing antibodies. To evaluate the influence of these factors we performed a variance analysis of the data. Mixing or sequential application alone had no significant influence on $\Delta \mathrm{IF}$ (Figure 9). The choice of the secondary reagent had a significant influence on $\Delta \mathrm{IF}$
$(P=0.029)$ but this effect was also dependent on the mode of application (binding between $\mathrm{F}\left(\mathrm{ab}^{\prime}\right)_{2}$ and mixing, $P=0.013)$. Explicitly, the $\mathrm{F}\left(\mathrm{ab}^{\prime}\right)_{2}$ fragment was superior when mixed but the whole molecule was better when applied sequentially (Figure 9). These data indicate that all combinations tested in this experiment gave a robust FRET signal. In principle, neither the choice of the secondary reagent nor the mode of application can be regarded as superior.

\section{Measurement of FRET in Human Glomus Tumors}

To test the feasibility to apply the technique to human material we examined resection specimens 

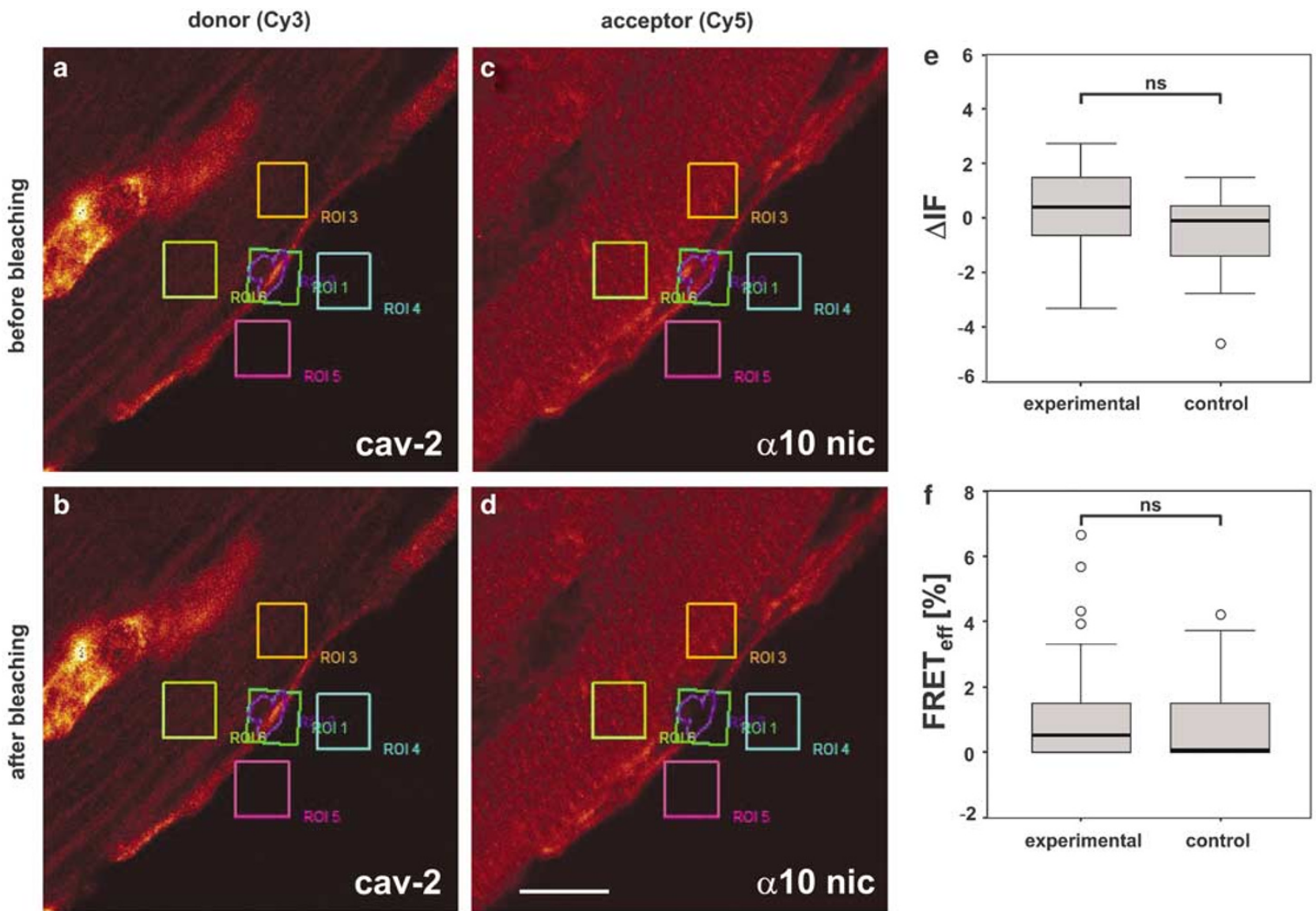

Figure 5 Examination of the FRET signal between the two non-interacting proteins $\alpha 10$ nicotinic acetylcholine receptor subunit and cav2 in the endocardium by indirect immunofluorescence and FRET in the rat heart. Images of donor (cav-2 labeled with Cy3-conjugated secondary antibody (a and $\mathbf{b})$ ) and acceptor ( $\alpha 10$ nicotinic acetylcholine receptor subunit ( $\alpha 10$ nic) labeled with Cy5-conjugated secondary antibody (c and d)) fluorescence. Cy5 was bleached in region of interest (ROI) 1 (compare ROI 1 (c and d)). (e) Changes in $\Delta \mathrm{IF}$, (f) same data expressed in $\mathrm{FRET}_{\text {eff }}, \mathrm{NS}=P>0.05$. Boxplots: percentiles 0, 25, median 75, 100, $\mathrm{O}=$ extreme values of data set. Secondary antibodies were sequentially incubated (Cy5-conjugated reagent first). Bar $=10 \mu \mathrm{m}$.
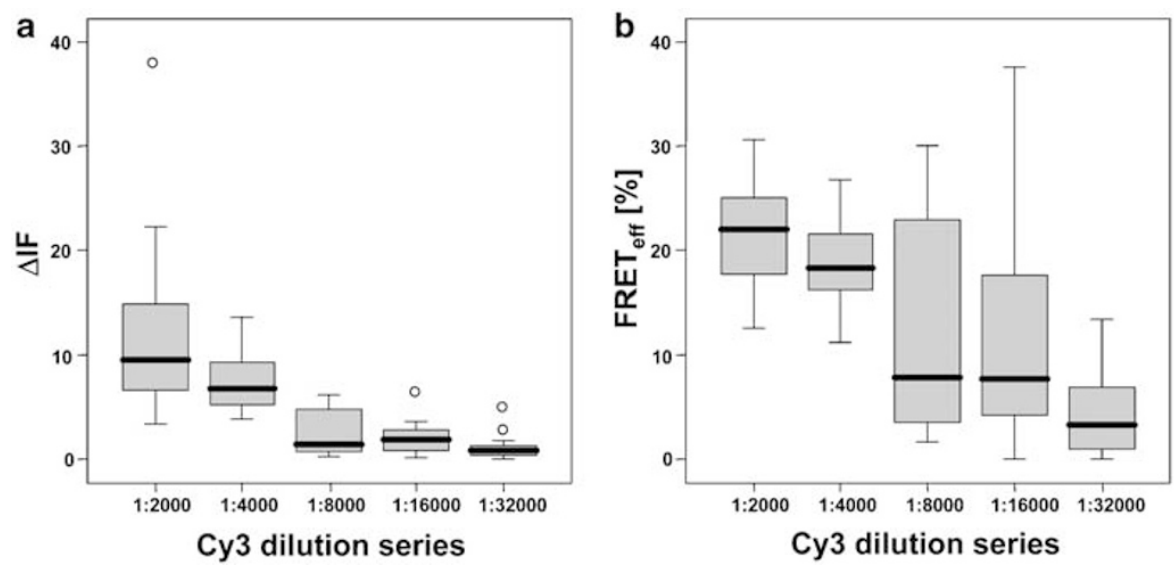

Figure 6 Simulation of crossreactivity by detection of cav-1 primary antibody with two secondary antibodies. Cy3-labeled secondary antibody was diluted from 1:2000 to 1:32 000 while the concentration of the Cy5-labeled secondary antibody was held constant. (a) Data

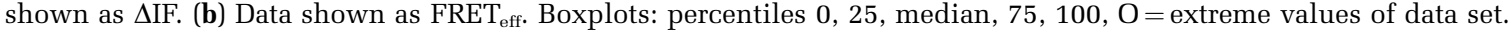

of glomus tumors that were previously fixed, cryoprotected and stored at $-80^{\circ} \mathrm{C}$. Glomus tumors showed the characteristic picture of tumor cells arranged in 'zellballen' separated by a highly vascular stroma (cf. Lack $^{14}$ ). The endothelial cells of these enlarged vessels exhibited distinct cav-1and cav-2 immunoreactivities. We were able to detect a robust FRET signal between cav-1 and cav2 in these specimens (Figure 10) (median $\Delta \mathrm{IF}$ experimental group: 4.81, control: 1.25, $P<0.001$ ). 
a

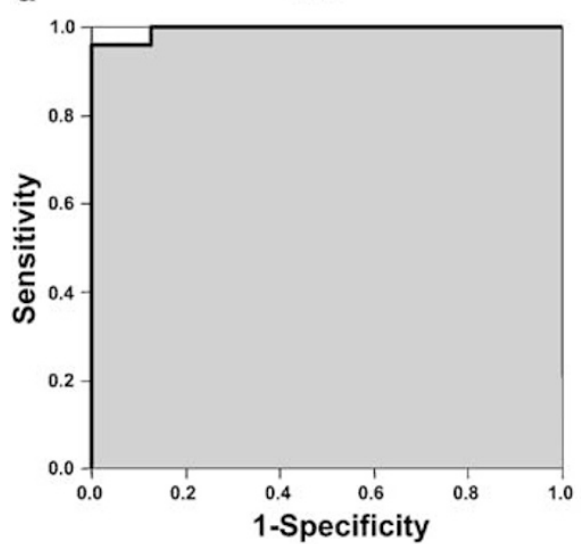

b FRET $_{\text {eff }}[\%]$

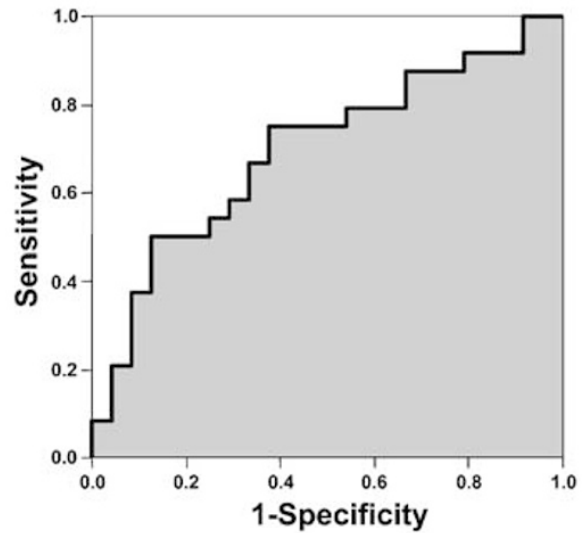

Figure 7 Comparison of $\mathrm{FRET}_{\text {eff }} v s \Delta \mathrm{IF}$ of the same data set to discriminate between the experimental and the control group by ROC curves. Boxplots of the data, see Figure 1 first boxplot (experimental group) and fourth boxplot (control). Area under the curve (AUC) shown in gray. (a) $\triangle \mathrm{IF} \mathrm{AUC}=0.995$. (b) $\mathrm{FRET}_{\text {eff }} \mathrm{AUC}=0.696$. Larger AUC indicates better discrimination between experimental vs control group.

a

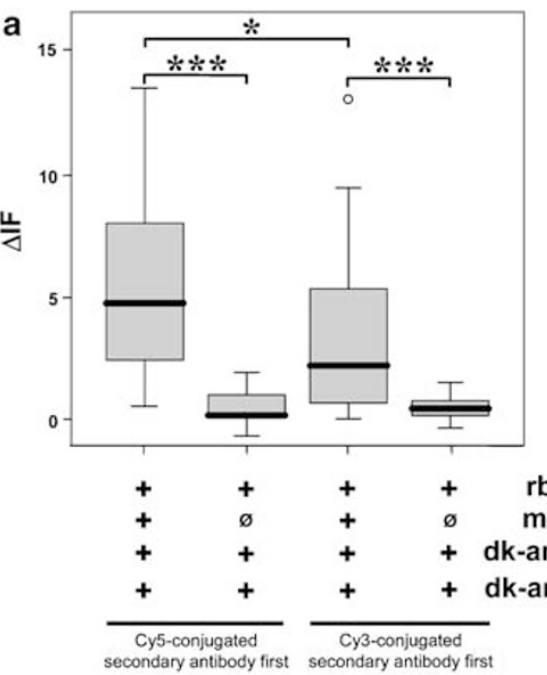

b

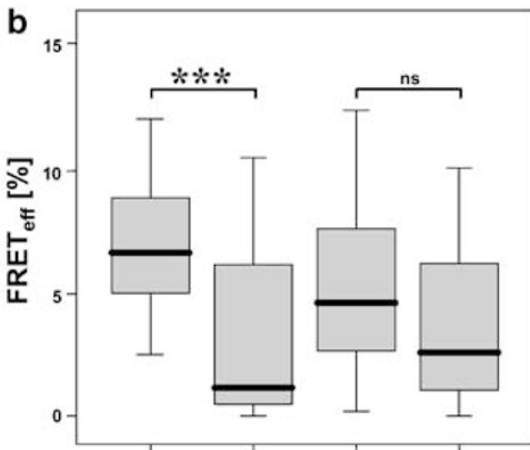

b-anti cav-1 ms-anti cav-2

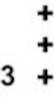

+
+
+
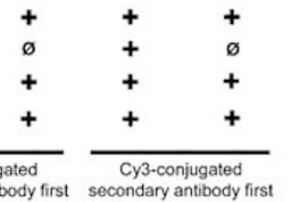

Figure 8 Influence of the incubation sequence of secondary antibodies. Cy5-conjugated secondary antibody first vs Cy3-conjugated secondary antibody first. ${ }^{*}=P<0.05,{ }^{* *}=P<0.001$, NS $=P>0.05$. (a) Data shown as $\Delta \mathrm{IF}$. (b) Data shown as FRET ${ }_{\text {eff }}$ Boxplots: percentiles 0,25 , median, $75,100, \mathrm{O}=$ extreme values of data set.

This demonstrates that the technique can be used to analyze human material that is derived from surgical procedures and is stored for extended times at $-80^{\circ} \mathrm{C}$.

\section{Discussion}

We were able to detect FRET in endothelial cells in tissue sections of rat and human specimens that were double labeled for cav-1 and cav-2 by indirect immunofluorescence. The interaction of both proteins was expected from biochemical experiments indicating that cav-1 and cav-2 form hetero-oligomers ${ }^{15,16}$ and that cav-2 needs cav-1 to be transported to the plasma membrane. ${ }^{7,8}$ Although the biochemical data predicted the close spatial association of both proteins in situ, this report shows for the first time this association in a specific cell type by light microscopy in tissue and provides the first description of cav-1 and cav-2 association in endothelial cells in human tissue.

Two additional experiments indicate that the detection of this interaction is specific. First, as expected, we did not detect a FRET signal between cav-2 and the $\alpha 10$ nicotinic acetylcholine receptor subunit. Second, we still detected an interaction between cav-1 and cav-2 when we switched the donor and the acceptor. Thus, detection of FRET does not depend on one particular set of secondary antibodies.

A major potential criticism of using indirect immunohistochemistry for FRET is the size of the antibody-antibody complexes used for detection. Could the size of the antibody-antibody complexes result in a FRET signal although the examined 

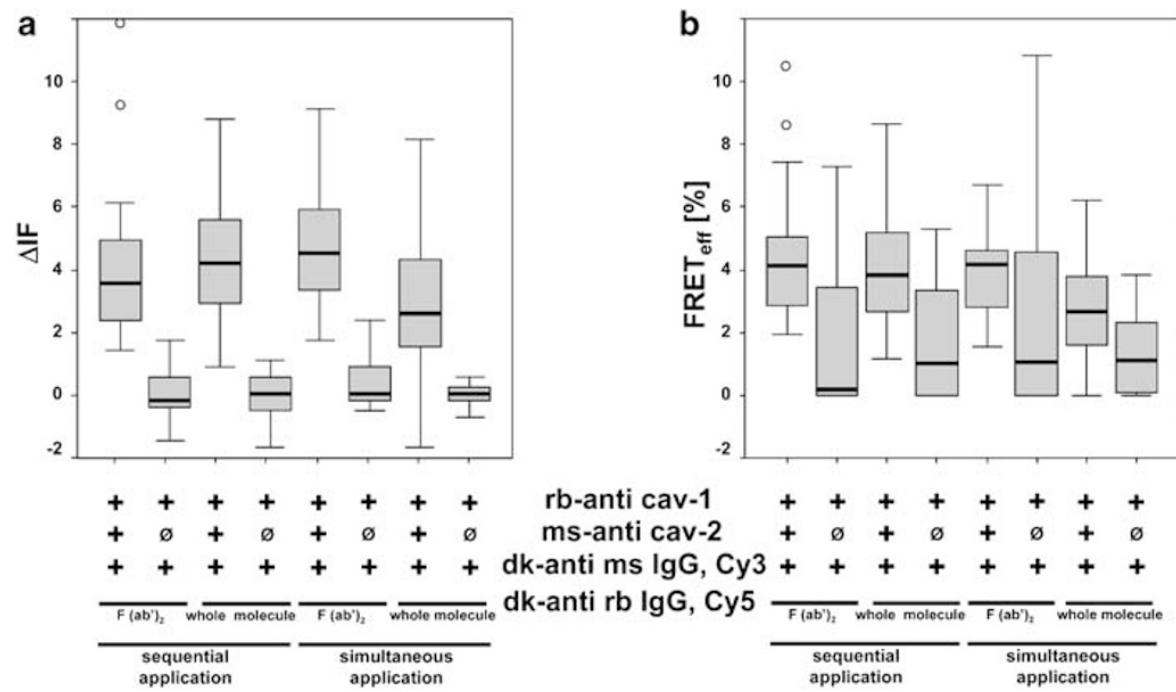

Figure 9 Comparison of Cy5-labeled whole antibody molecule vs Cy5-labeled $\mathrm{F}\left(\mathrm{ab}^{\prime}\right)_{2}$ fragment and comparison of incubating the Cy5conjugated secondary reagent first vs mixing both secondary antibodies. (a) Data shown as $\Delta \mathrm{IF}$. (b) Data shown as FRET eff. Boxplots: percentiles 0,25 , median, $75,100, \mathrm{O}=$ extreme values of data set.
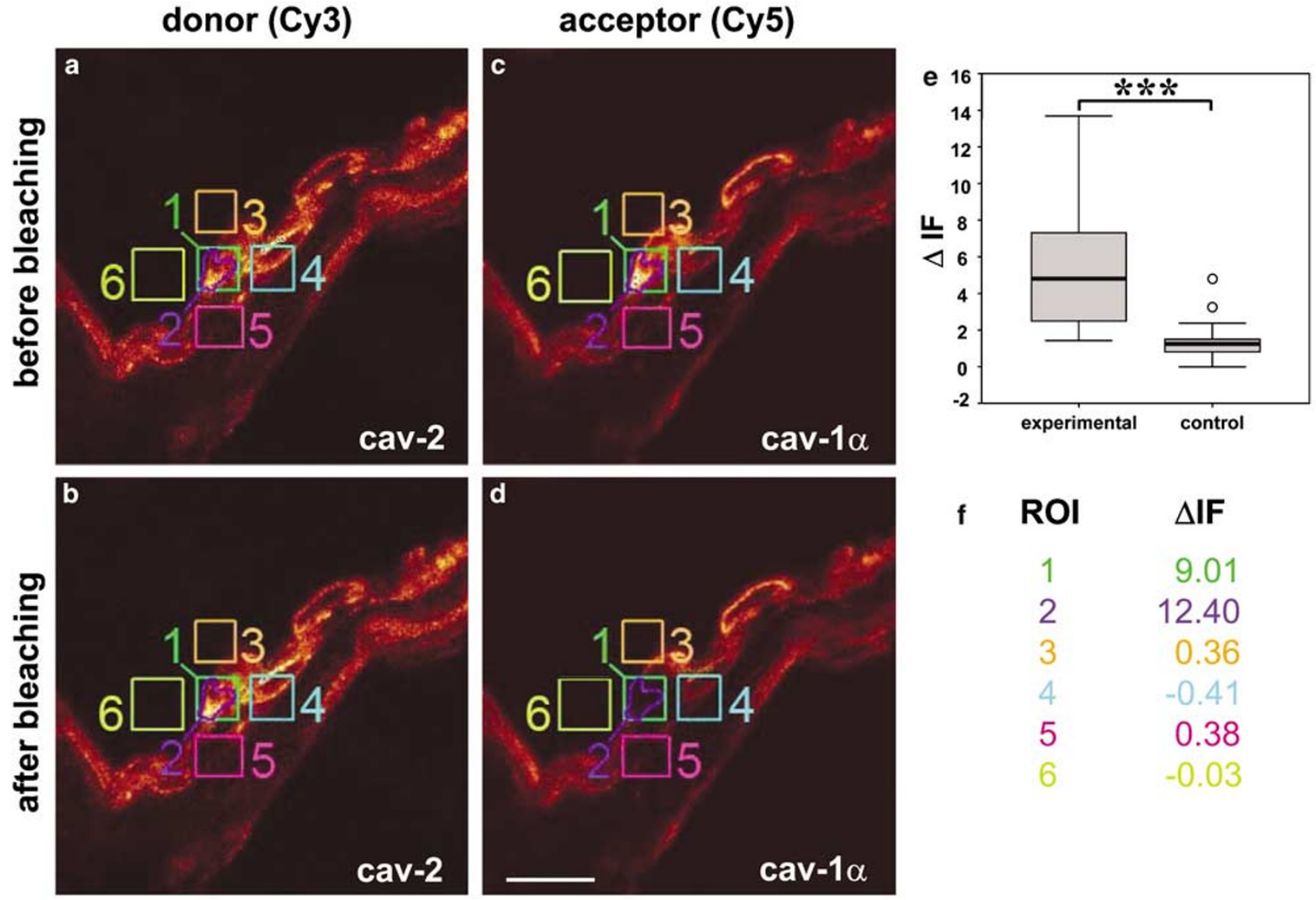

Figure 10 Detection of close association of cav-1 and cav-2 by indirect immunofluorescence and FRET in human glomus tumors. Images of donor (cav-2 labeled with Cy3-secondary antibody (a and b)) and acceptor (cav-1 $\alpha$ labeled with Cy5-secondary antibody (c and d)). Cy5 was bleached in ROI 1 (compare ROI 1 (c and d)). (e) Data analysis. ${ }^{* * *}=P<0.001$. (f) Changes in $\Delta$ IF in each of the ROI. ROI 1: bleached area (compare (c and d)). ROI 2: region of endothelial cell in bleached area. ROI 3-6: control areas outside the bleached area. Boxplots: percentiles 0,25 , median, $75,100, \mathrm{O}=$ extreme values of data set. $\mathrm{Bar}=10 \mu \mathrm{m}$.

proteins are further than $10 \mathrm{~nm}$ apart? The $\mathrm{FRET}_{\text {eff }}$ we measured was in the range of $4-15 \%$ which would correspond to a distance of about $9.2-7.2 \mathrm{~nm}$ between Cy3 and Cy5 (Förster constant $R_{0}=5.4 \mathrm{~nm}$ for Cy3 and $\mathrm{Cy} 5{ }^{17}$ ). Taking into consideration that the length of an antibody is about $10 \mathrm{~nm}^{18}$ the 
maximal size of a primary antibody-secondary antibody complex is about $20 \mathrm{~nm}$ if they are linearly aligned. The maximal distance of two antibodyantibody complexes bound to cav- 1 and cav- 2 could be expected to be about $40 \mathrm{~nm}$ plus the distance between the binding sites of the antibodies and still give a FRET signal (Figure 11a). If this scenario holds true, a FRET signal would still be expected to occur even if cav-1 and cav-2 were about $50 \mathrm{~nm}$ apart which is considerably more than the suggested $10 \mathrm{~nm}$. However, several theoretical as well as practical considerations favor a scenario in which the two proteins are in fact clearly $<50 \mathrm{~nm}$ apart (Figure 11b). First, the fluorophores are most likely not exclusively located at the outermost part of the Fc part of the antibody. Second, a geometrical scenario that both secondary antibodies bind to the distal part of the $F_{C}$ fragments and that these antibody-antibody complexes are lying flat on the
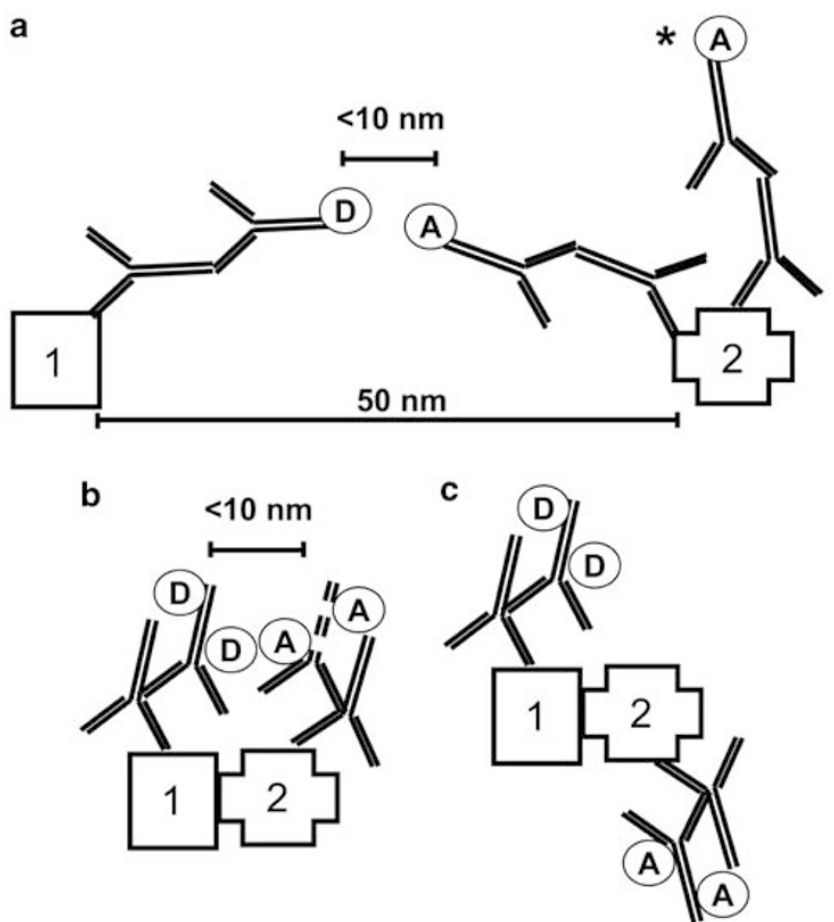

Figure 11 Possible spatial arrangements of antigen-antibody complexes. The spatial arrangement in (a) in which the antigens 1 and 2 are $50 \mu \mathrm{m}$ apart and a FRET signal occurs is highly unlikely since the secondary antibodies have to bind to the tip of the Fc fragment of the primary antibody, the fluorophores have to be located at the tips of the secondary antibodies and both antibody-antigen complexes have to be aligned exactly. A much more likely configuration is that at least one of the antigenantibody complexes are arranged differently (asterisk) and no FRET occurs. If FRET is detected, a configuration as shown in (b) can be assumed in which both proteins 1 and 2 are in close apposition, the secondary antibodies do not bind to the tip of the FC fragment of the primary antibody and the fluorophores are not located exclusively at the tip of the secondary antibody. In this configuration, the absence of an Fc fragment of a secondary antibody (dashed lines) does not have a significant impact on the FRET signal. (c) If the location of antibody binding is unfavorable FRET does not occur, even if the proteins 1 and 2 interact. caveolar membrane is highly unlikely. Third, if they did, they would have to be located in such a way that two Fc parts of the secondary antibodies point directly towards each other (cf. Figure 11a). Most important, our data indicate that use of a $\mathrm{F}\left(\mathrm{ab}^{\prime}\right)_{2}$ fragment instead of a whole antibody molecule does not reduce the FRET signal as it would be expected if the antibodies bridged a larger distance. It is therefore highly unlikely that FRET signals based on indirect immunofluorescence occur with proteins that are not in close spatial association. In contrast, it is possible that although proteins interact, the method gives no FRET signal because the position of the antibody-antibody complexes towards each other is unfavorable.

Since cav-1 and cav-2 are present in multiple copies per caveola, sterical hindrance might also reduce the binding of secondary antibodies, resulting in lower FRET. Indeed, we observed higher FRET $_{\text {eff }}$ if the Cy5-conjugated secondary antibody was incubated prior to the Cy3-labeled antibody compared to the incubation of the Cy3-labeled antibody first. This indicates (1) that the secondary antibodies hinder each other's binding, and (2) that binding of larger amounts of acceptor (Cy5) can be favorable. A further result pointing in this direction is the higher FRET signal when the polyclonal cav-1 antibody was labeled with Cy5-conjugated antibody as compared to the labeling of the monoclonal cav-2 antibody with a Cy5-conjugated secondary antibody. Since polyclonal antibodies can bind to more than one epitope, the chance to bind to a protein is higher than with a monoclonal antibody. Thus, this could explain why we saw higher FRET signals when we labeled the polyclonal antibody with the acceptor (Cy5). Although we did see a clear difference between the application of Cy5- vs Cy3-conjugated secondary antibody first, we did not detect clear differences between Cy5 application first and simultaneous application of the secondary antibody. This indicates that simultaneous application of the secondary antibodies can result in sufficient amount of binding of acceptor-labeled antibodies to result in good FRET signal.

That acceptor bleaching is very well suited to detect FRET even if only small amounts of donor molecules are present is underpinned by our dilution experiments in which very small amounts of Cy3-conjugated antibody that are not seen microscopically still resulted in measurable FRET if Cy5 was present in large amounts. In our experiments, the only limiting factor to detect FRET is the stability of the setup and the bleaching of the donor during acceptor bleaching and image acquisition which can obscure small increases in donor fluorescence.

Surprisingly, we measured a FRET signal in control experiments when that primary antibody was omitted that was normally labeled with the Cy3labeled secondary antibody and both secondary antibodies were applied. Although secondary anti- 
bodies were crossadsorbed to the serum proteins of the other primary antibody's host species to reduce crossreactivity, the most likely explanation for this phenomenon is a minimal, yet measurable crossreactivity of the Cy3-conjugated secondary antibody. This is supported by the fact that in an experiment where both secondary antibodies were directed against the same primary antibody, FRET could be detected even if the concentration of the Cy3conjugated secondary antibody was diluted up to the point that no Cy3 labeling was microscopically visible. Since FRET $_{\text {eff }}$ is the percentage of fluorescence increase, small changes in $\Delta \mathrm{IF}$ result in large $\mathrm{FRET}_{\text {eff }}$ if the initial Cy3 fluorescence intensity is low. This explains the rather large FRET $_{\text {eff }}$ in control sections in contrast to the small increase in $\Delta \mathrm{IF}$. Furthermore, the FRET signal measured under experimental conditions has to be interpreted as a mixture of two causes, first, the $\mathrm{FRET}_{\text {eff }}$ resulting from labeling cav-1 and cav-2, and second, the crossreactivity of both secondary antibodies. Taking into consideration that crossreactivity of secondary antibodies results in binding of two antibodies to the same primary antibody, it should be expected that the resulting $\mathrm{FRET}_{\text {eff }}$ is as high as or even higher than under experimental conditions where the secondary antibodies bind to different primary antibodies. Therefore, solely based on theoretical reasons $\mathrm{FRET}_{\text {eff }}$ shall not be a reliable parameter to detect an association of two proteins by indirect immunohistochemistry and FRET. Indeed, statistical comparison of the ability of FRET $\mathrm{Tff}_{\text {eff }}$ and $\mathrm{IF}$ to discriminate experimental and control conditions clearly shows that $\Delta \mathrm{IF}$ is more sensitive and more specific to discriminate control from experimental conditions. We therefore propose $\Delta \mathrm{IF}$ as the preferred parameter for measuring FRET in this setup provided both the control and the experimental conditions are measured under identical CLSM settings.

In addition to crossreactivity, we could identify another possible source that interferes with FRET measurements. We experienced that in some experiments $\Delta \mathrm{IF}$ changed not only in the area that was bleached but also in areas outside the bleached ROI. We found that air movements that result from the air-conditioning system or from opening the door of the microscope room often also resulted in changes of $\Delta \mathrm{IF}$ outside the bleached area. Therefore, it is mandatory to control for stability of the imaging system by measuring ROI outside the bleached area to validate if the measured changes indeed result from FRET or from movements of the specimen in $z$-direction.

If controlled properly, double-labeling immunofluorescence and FRET-CLSM hold strong promises to examine protein-protein association in tissue sections and can also be successfully used to examine such interactions in human material. Since the method is inexpensive, quick and requires only a standard CLSM that is accessible in most institu- tions, it opens up the possibility to gain insight in protein-protein interactions in tissue sections in normal as well as in human material derived from surgical procedures.

\section{Acknowledgements}

We thank Karola Michael for expert technical help with the figures and Dr RM Bohle for critically reading the manuscript. The histopathological diagnosis of glomus tumors was conducted at the Institut für Pathologie, Universitätsklinikum Giessen. This study was supported by the Deutsche Forschungsgemeinschaft (Sonderforschungsbereich 547, C1; Graduiertenkolleg 534 both to WK) and a young scientist's grant from the Fachbereich Medizin der the Justus-Liebig-Universität Giessen to PK.

\section{References}

1 Garini Y, Vermolen BJ, Young IT. From micro to nano: recent advances in high-resolution microscopy. Curr Opin Biotechnol 2005;16:3-12.

2 Jares-Erijman EA, Jovin TM. FRET imaging. Nat Biotechnol 2003;21:1387-1395.

3 Kenworthy AK. Imaging protein-protein interactions using fluorescence resonance energy transfer microscopy. Methods 2001;24:289-296.

4 Sharma N, Hewett J, Ozelius LJ, et al. A close association of torsin A and alpha-synuclein in Lewy bodies: a fluorescence resonance energy transfer study. Am J Pathol 2001;159:339-344.

5 Mills JD, Stone JR, Rubin DG, et al. Illuminating protein interactions in tissue using confocal and twophoton excitation fluorescent resonance energy transfer microscopy. J Biomed Opt 2003;8:347-356.

6 Razani B, Woodman SE, Lisanti MP. Caveolae: from cell biology to animal physiology. Pharmacol Rev 2002; 54:431-467.

7 Capozza F, Cohen AW, Cheung MW, et al. Musclespecific interaction of caveolin isoforms: differential complex formation between caveolins in fibroblastic vs muscle cells. Am J Physiol Cell Physiol 2005;288: C677-C691.

8 Drab M, Verkade P, Elger M, et al. Loss of caveolae, vascular dysfunction, and pulmonary defects in caveolin-1 gene-disrupted mice. Science 2001;293: 2449-2452.

9 Dvorakova M, Lips KS, Brüggmann D, et al. Developmental changes in the expression of nicotinic acetylcholine receptor alpha-subunits in the rat heart. Cell Tissue Res 2005;319:201-209.

10 Brüggmann D, Lips KS, Pfeil U, et al. Rat arteries contain multiple nicotinic acetylcholine receptor alpha-subunits. Life Sci 2003;72:2095-2099.

11 Lips KS, Pfeil U, Kummer W. Coexpression of alpha 9 and alpha 10 nicotinic acetylcholine receptors in rat dorsal root ganglion neurons. Neuroscience 2002;115: $1-5$.

12 Maurer WHL, Lehmacher W. Multiple comparisons in drug clinical trials and preclinical assays: a priori ordered hypothesis. In: Vollmar J (ed). Biometrie in der 
Chemisch-Pharmazeutischen Industrie. Fischer: Stuttgart, 1995, pp 3-18.

13 Hanley JA, McNeil BJ. The meaning and use of the area under a receiver operating characteristic (ROC) curve. Radiology 1982;143:29-36.

14 Lack EE. Pathology of Adrenal and Extra-Adrenal Paraganglia. W.B. Saunders: Philadelphia, USA, 1994.

15 Das K, Lewis RY, Scherer PE, et al. The membranespanning domains of caveolins-1 and -2 mediate the formation of caveolin hetero-oligomers. Implications for the assembly of caveolae membranes in vivo. J Biol Chem 1999;274:18721-18728.
16 Scherer PE, Lewis RY, Volonte D, et al. Cell-type and tissue-specific expression of caveolin-2. Caveolins 1 and 2 co-localize and form a stable hetero-oligomeric complex in vivo. J Biol Chem 1997; 272:29337-29346.

17 Sabanayagam CR, Eid JS, Meller A. Using fluorescence resonance energy transfer to measure distances along individual DNA molecules: corrections due to nonideal transfer. J Chem Phys 2005;122:061103.

18 Harris LJ, Skaletsky E, McPherson A. Crystallographic structure of an intact IgG1 monoclonal antibody. J Mol Biol 1998;275:861-872. 